\title{
Clinical Application and Psychometric Properties of a Swedish Translation of the Abbreviated Profile of Hearing Aid Benefit
}

\author{
K. Jonas Brännström, $\mathrm{PhD}^{1} \quad$ Ketty Andersson, $\mathrm{PhD}^{1} \quad$ Olof Sandgren, $\mathrm{PhD}^{1} \quad$ Susanna Whitling, $\mathrm{PhD}^{1}$ \\ ${ }^{1}$ Logopedics, Phoniatrics and Audiology, Department of Clinical \\ Sciences - Lund, Lund University, Lund, Sweden \\ Address for correspondence K. Jonas Brännström, PhD, \\ jonas.brannstrom@med.lu.se \\ J Am Acad Audiol 2020;31:656-665.
}

\begin{abstract}
Background: The impact of hearing loss on the individual and his/her everyday life can be assessed using questionnaires with the purpose to improve rehabilitation quality. The Abbreviated Profile of Hearing Aid Benefit (APHAB) can be used to evaluate disability in everyday life associated with hearing loss. Previous studies have examined APHAB outcomes in sensorineural hearing loss and we do not know whether the type of hearing loss influence questionnaire outcomes.

Purpose: The purpose was to evaluate the psychometric properties of a Swedish translation of the APHAB and the influence of demographic variables on the outcome in a clinical sample.

Research Design: A descriptive, cross-sectional study in a clinical sample.

Study Sample: Forty-eight participants with no hearing aid experience seeking audiological rehabilitation for the first time. These participants represented different degrees of hearing loss and three types of hearing loss: monaural mixed, binaural mixed, and binaural sensorineural hearing loss.

Data Collection and Analysis: Pure-tone audiometry was conducted and the participants completed the unaided APHAB during their first appointment at the clinic. Psychometric properties of the questionnaire were examined and the influence of age, gender, type of hearing loss, and degree of hearing loss on APHAB scores were studied. Results: The psychometric properties indicate high test-retest reliability but there seems to be some potential issues with the properties of the reverberation (RV) subscale. The items from the RV subscale failed to load as a separate component and the internal consistency of the subscale improved by removing four items (items 1,9 , 11 , and 16). With few exceptions, APHAB scores were not influenced by age, gender, or type of hearing loss. APHAB scores were generally influenced by degree of hearing loss

Keywords

- questionnaires

- auditory rehabilitation

- first time

in both the best and the worst ear.

Conclusion: This Swedish version of the APHAB can be additionally improved by addressing the inconsistencies found in the RV subscale by rephrasing or removing some items. The degree of hearing loss has some influence on questionnaire outcomes but not age, gender, and type of hearing loss.
\end{abstract}

received

December 6, 2019

accepted after revision

February 3, 2020

published online

December 9, 2020
(C) 2020. American Academy of

Audiology. All rights reserved.

Thieme Medical Publishers, Inc.,

333 Seventh Avenue, 18th Floor,

New York, NY 10001, USA
DOI https://doi.org/

10.1055/s-0040-1718702.

ISSN 1050-0545. 
The impact of hearing loss on verbal communication and the ability to auditorily perceive environmental sounds may lead to the experience of disability in the form of participation restrictions and activity limitations. Individuals with hearing loss may experience poorer quality of life due to withdrawal caused by these participation restrictions and activity limitations. ${ }^{1-3}$ Audiological rehabilitation aims to ameliorate the impact of hearing loss. This is often achieved by fitting listening devices and improving communication strategies. When beginning rehabilitation, it is therefore valuable to identify areas of importance for the individual to guide the clinician. ${ }^{4}$ The impact of hearing loss on an individual and their everyday life can be assessed at the clinic using questionnaires to improve rehabilitation quality. ${ }^{5,6}$ The use of questionnaires is also a way to involve patients in their rehabilitation process. Knowing which factors may influence questionnaire responses can further promote rehabilitation quality and outcomes. ${ }^{5,7}$

The Abbreviated Profile of Hearing Aid Benefit (APHAB) was developed by Cox and Alexander. ${ }^{4}$ It is a disability-based self-assessment questionnaire that can be used to quantify subjective disability in everyday life associated with hearing loss and also to measure the effect of hearing aid intervention on subjective disability. ${ }^{4}$ Here, we use the term disability as a composite expression for participation restrictions and activity limitations. In line with the International Classification of Functioning (ICF), an individual's capacity for participation in different parts of life, is decided by external and internal factors, by body functions and structure, as well as any disorder, for example, impaired hearing. ${ }^{8}$ APHAB can be used to assess the frequency of communication problems and problems with environmental noises with (aided) and without (unaided) amplification and the difference between these two listening conditions (benefit). ${ }^{9}$ The unaided APHAB has previously been used to explore associations with pure-tone average (PTA) and performance on speech recognition tests in subjects with different degrees of binaural sensorineural hearing loss. ${ }^{9}$ Higher APHAB scores were found to be correlated to poorer PTA for subscales ease of communication (EC), background noise (BN), and reverberation (RV), but not for aversiveness (AV) toward environmental sounds. ${ }^{9}$ That AV was not associated with PTA was explained by the fact that the AV subscale relates to unpleasantness of environmental sounds while PTA relates to detecting soft sounds. ${ }^{9}$ Higher self-reported disability on the unaided $A P H A B$ are associated with increased probability of hearing loss, especially in higher frequencies. ${ }^{10}$ An additionally abbreviated Norwegian version of the APHAB (where number of items were reduced and response options were changed) has been used to assess the relationship between unaided responses and some demographic variables. ${ }^{11}$ They found that degree of hearing loss (PTA) influenced responses but they found no differences between male and female participants or between experienced and first-time hearing aid users.

The psychometric properties of the APHAB have been evaluated for the English version in experienced hearing aid users. ${ }^{4}$ The findings indicate satisfactory internal consistency of the separate subscales. Test-retest values indicate that a change in ratings on a subscale for an individual participant should exceed approximately 17 to $26 \%$ to be significant. ${ }^{4} \mathrm{~A}$ Swedish translation of the APHAB exist ${ }^{12}$ and some psychometric properties have been reported. ${ }^{12,13}$ For experienced hearing aid users with mild to moderate hearing loss, Arlinger et $\mathrm{al}^{12}$ found mean scores for the four subscales that were marginally higher than those reported by Cox and Alexander. ${ }^{4}$ Arlinger et $\mathrm{al}^{12}$ reported very minor test-retest differences (about $\pm 2 \%$ units). Berninger and Karlsson ${ }^{13}$ found mean scores for first time hearing aid users similar to those reported by both Cox and Alexander ${ }^{4}$ and Arlinger et al. ${ }^{12}$

The present study evaluates disability in everyday life associated with hearing loss in patients seeking audiological rehabilitation for the first time using a Swedish translation of APHAB. ${ }^{4}$ The psychometric properties of the questionnaire are comprehensively examined and the influence of some demographic variables on outcomes is explored. The aim was to evaluate self-assessed disability in everyday life associated with hearing loss using a Swedish translation of the APHAB in patients seeking audiological rehabilitation for the first time. This study also explores the psychometric properties of the Swedish version of the questionnaire and how age, gender, type of hearing loss, and degree of hearing loss influence its outcome.

\section{Materials and Method}

\section{Participants}

Fifty-five participants with no previous experience of hearing aids were initially recruited to the study. Participants were recruited from two separate audiological clinics in the region Skåne in the southernmost part of Sweden. Since the study is mainly descriptive, no control group was recruited. As assessed by clinical audiologists, participants were eligible candidates for receiving their first hearing aid(s). To be included, the participants had to be Swedish speaking (not necessarily native speakers), have a PTA equal to or worse than $25 \mathrm{~dB}$ hearing level $(\mathrm{HL})$ in their worst ear for frequencies $0.5,1.0,2.0$, and $4.0 \mathrm{kHz}$, and provided responses on $80 \%$ or more of the items in each APHAB subscale. For the purposes of the present study, audiometry was conducted using either a MADSEN Conera audiometer or an Otometrics Aurical audiometer together with Telephonics TDH-39P supra-aural earphones and Radioear B71 bone conductor. The complete equipment set-up was calibrated in accordance with IEC 60318-3, ISO 389-1, and ISO 389-3. ${ }^{14-16}$ Pure tones (frequencies and test order 1.0, 1.5, 2.0, 3.0, 4.0, 6.0, 8.0, 0.5, 0.25 , and $0.125 \mathrm{kHz}$ ) of 1 to 2 seconds duration were used. The air conduction (frequencies and test order 1.0, 1.5, 2.0, $3.0,4.0,6.0,8.0,0.5,0.25$, and $0.125 \mathrm{kHz}$ ) and bone conduction audiometry (frequencies and test order 1.0, 1.5, 2.0, 3.0, $4.0,0.5$, and $0.25 \mathrm{kHz}$ ) were performed in accordance with ISO $8253-1^{17}$ using the manual ascending technique ($10 \mathrm{~dB} /+5 \mathrm{~dB})$. The Regional Ethical Review Board in Lund approved the project (2010/240) and informed written consent was received from all participants.

Out of 55 recruits, 53 completed the study. However, an additional five subjects did not provide responses on $80 \%$ or 


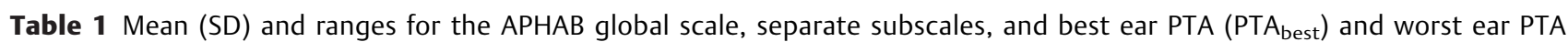
$\left(\mathrm{PTA}_{\text {worst }}\right)$ for subjects with monaural mixed hearing loss $(n=12)$, binaural mixed hearing loss $(n=13)$, binaural sensorineural hearing loss $(n=28)$, and all subjects together $(n=48)$

\begin{tabular}{|l|l|l|l|l|l|l|l|l|l|l|l|l|l|}
\hline & \multicolumn{4}{l|}{ Monaural mixed } & \multicolumn{2}{l|}{ Binaural mixed } & \multicolumn{2}{l|}{ Binaural sensorineural } & \multicolumn{2}{ll}{ All subjects } \\
\hline Variable & Mean (SD) & Range & \multicolumn{2}{l|}{ Mean (SD) } & \multicolumn{2}{l|}{ Range } & \multicolumn{2}{l|}{ Mean (SD) } & \multicolumn{2}{l|}{ Range } & \multicolumn{2}{l|}{ Mean } & Range \\
\hline Global & 44.6 & $(15.0)$ & $28.5-73.2$ & 54.6 & $(13.0)$ & $33.1-75.4$ & 49.2 & $(15.2)$ & $22.3-75.9$ & 49.4 & $(14.8)$ & $22.3-75.9$ \\
\hline EC & 33.3 & $(23.5)$ & $12.3-91.8$ & 51.5 & $(21.7)$ & $19.0-82.2$ & 40.2 & $(18.0)$ & $4.7-78.7$ & 41.3 & $(21.0)$ & $4.7-91.8$ \\
\hline RV & 43.7 & $(18.7)$ & $18.8-70.0$ & 56.4 & $(16.1)$ & $32.4-85.0$ & 51.8 & $(20.6)$ & $22.8-93.0$ & 50.9 & $(19.3)$ & $18.8-96.0$ \\
\hline BN & 57.0 & $(17.1)$ & $37.5-91.0$ & 55.5 & $(14.4)$ & $32.4-78.8$ & 55.9 & $(17.2)$ & $12.7-95.0$ & 56.1 & $(16.2)$ & $12.7-95.0$ \\
\hline AV & 40.8 & $(25.6)$ & $8.3-99.0$ & 29.9 & $(18.3)$ & $4.7-70.5$ & 18.8 & $(16.5)$ & $1.0-64.2$ & 27.1 & $(21.2)$ & $1.0-99.0$ \\
\hline PTA $_{\text {best }}$ & 13.3 & $(6.8)$ & $5-24$ & 39.0 & $(10.3)$ & $26-61$ & 36.0 & $(9.4)$ & $15-60$ & 31.1 & $(13.7)$ & $5-61$ \\
\hline PTA $_{\text {worst }}$ & 46.5 & $(6.1)$ & $39-59$ & 67.2 & $(15.0)$ & $41-94$ & 45.0 & $(13.1)$ & $25-84$ & 51.0 & $(15.3)$ & $25-94$ \\
\hline
\end{tabular}

Abbreviations: APHAB, Abbreviated Profile of Hearing Aid Benefit; AV, aversiveness; BN, background noise; EC, ease of communication; PTA, puretone average; RV, reverberation; SD, standard deviation.

more of the items in each APHAB subscale and were therefore excluded. Thus, 48 participants were included in the analysis (31 men and 17 women; mean age 63.0 years, standard deviation [SD] 14.5 years, range 27-85 years). Based on their audiogram, the participants were pooled into three groups. The first group, monaural mixed hearing loss $(n=12)$, had a better ear PTA equal to or better than $25 \mathrm{~dB}$ HL. In their poorer ear, they had a hearing loss where the bone conduction PTA was poorer than $25 \mathrm{~dB}$ HL and the average air-bone gap was equal to or exceeded $15 \mathrm{~dB}$. The second group, binaural mixed hearing loss $(n=12)$, had bone conduction PTA poorer than $25 \mathrm{~dB}$ HL in both better and poorer ear. The third group, binaural sensorineural hearing loss $(n=24)$, had a better ear air conduction PTA poorer than $25 \mathrm{~dB}$ HL and an average air-bone gap less than $15 \mathrm{~dB}$ in both ears. Average PTA for best and worst ears for all participants together and the three groups separately are shown in - Table 1 .

To be able to assess test-retest reliability of the unaided APHAB, a subsample of 28 participants (21 men and 7 women; mean age 59.9 years, SD 14.3, range 27-85 years) completed the questionnaire at two sessions. These participants represented the three groups: monaural mixed hearing loss $(n=10)$, binaural mixed hearing loss $(n=11)$, and binaural sensorineural hearing loss $(n=7)$. Average PTA for best and worst ears for the three groups based on the subsample was very similar to those of the whole sample.

\section{Abbreviated Profile of Hearing Aid Benefit}

APHAB consists of 24 items. ${ }^{4,9}$ Each item consists of a statement such as "Traffic noises are too loud." Seven responses are possible for each item; never (1\%), seldom (12\%), occasionally (25\%), half-the-time (50\%), generally (75\%), almost always (87\%), and always (99\%) and a higher score indicates more difficulties. Both response options and percentages are visible on the questionnaire. The responses on the questionnaire are scored in four subscales pertaining to EC (items 4, 10,12, 14, 15, and 23), BN (items 1, 6, 7, 16, 19, and 24), RV (items 2, 5, 9, 11, 18, and 21), and AV toward environmental sounds (items $3,8,13,17,20$, and 22). ${ }^{4}$ The separate items are shown in - Table 2 for the English version.
The subscale scores are calculated as the mean of the items in each subscale. A global scale score is also calculated as the mean scores for items in the three first subscales (i.e., EC, RV, and BN). As previously stated, for valid global scale and subscale scores ( $E C, B N, R V$, and $A V$ ) responses were required on $80 \%$ of the items or more. Participants responded to the questionnaire on paper using a pen after being verbally informed about how to complete the questionnaire. Reversed items were inverted prior to analysis.

\section{Overall Procedures}

Otoscopy and pure-tone audiometry was conducted and the participants completed the unaided Swedish APHAB during their first appointment at the clinic. As all participants were about to receive hearing aids, earmold impressions were also made when applicable and information on the effects of hearing loss and communication strategies were provided by an audiologist. The participants taking part in the test-retest part of the study participated in two sessions at the clinic, separated by approximately 4 weeks. During this intermission, no hearing aids were used.

\section{Results}

The demographic (independent) variables consisted of age, gender, best ear PTA (PTA best $)$, worst ear PTA (PTA worst $\left._{\text {, }}\right)$, and type of hearing loss (monaural mixed, binaural mixed, and binaural sensorineural). The dependent variables were APHAB global scale and the separate subscales (EC, BN, RV, and $A V$ ). When possible, nonparametric tests were used since responses on the APHAB were on ordinal scale. A two-tailed $\alpha$ level of 0.05 was considered statistically significant. In the case of multiple comparisons, Bonferroni corrections were used.

\section{Psychometric Properties}

The mean scores, SDs, and ranges for the APHAB global scale and four subscales are presented in - Table 1 and for individual items in - Table $\mathbf{2}$. The interitem correlations for the items in the global score and for each subscale indicated that 
Table 2 Mean (SD) and ranges for separate APHAB items

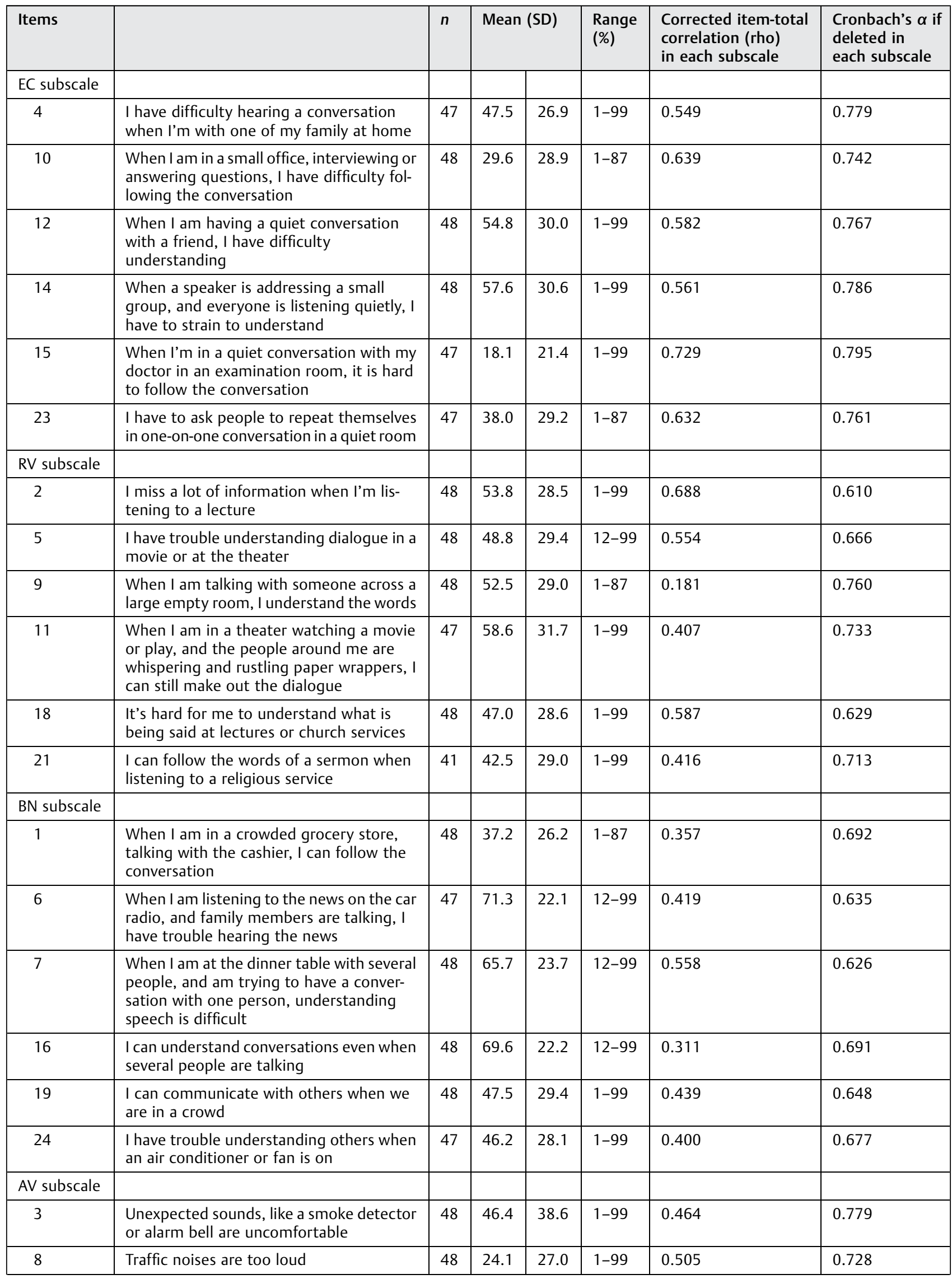


Table 2 (Continued)

\begin{tabular}{|c|l|l|l|l|l|l|l|}
\hline Items & & $n$ & Mean (SD) & $\begin{array}{l}\text { Range } \\
(\%)\end{array}$ & $\begin{array}{l}\text { Corrected item-total } \\
\text { correlation (rho) } \\
\text { in each subscale }\end{array}$ & $\begin{array}{l}\text { Cronbach's } \alpha \text { if } \\
\text { deleted in } \\
\text { each subscale }\end{array}$ \\
\hline 13 & $\begin{array}{l}\text { The sounds of running water, such as a } \\
\text { toilet or shower, are uncomfortably loud }\end{array}$ & 48 & 12.5 & 22.4 & $1-99$ & 0.523 & 0.736 \\
\hline 17 & $\begin{array}{l}\text { The sounds of construction work are un- } \\
\text { comfortably loud }\end{array}$ & 47 & 28.6 & 27.4 & $1-99$ & 0.661 & 0.674 \\
\hline 20 & $\begin{array}{l}\text { The sound of a fire engine siren close by is } \\
\text { so loud that I need to cover my ears }\end{array}$ & 48 & 25.4 & 32.0 & $1-99$ & 0.624 & 0.673 \\
\hline 22 & $\begin{array}{l}\text { The sound of screeching tires is uncom- } \\
\text { fortably loud }\end{array}$ & 46 & 23.4 & 28.5 & $1-99$ & 0.693 & 0.680 \\
\hline
\end{tabular}

Abbreviations: APHAB, Abbreviated Profile of Hearing Aid Benefit; AV, aversiveness; BN, background noise; EC, ease of communication; RV, reverberation; SD, standard deviation.

Note: Also shown are the corrected item-total correlations (Spearman's rho) and Cronbach's $\alpha$ if item deleted for the items in the separate subscales. Cronbach's alphae are shown for the separate subscales.

not all items in a subscale were correlated. The interitem correlations (Spearman's rho) for the global scale ranged between $-0.220(p=0.166)$ and $0.739(p<0.001)$. The interitem correlations (rho) for EC ranged between 0.310 $(p=0.034)$ and $0.689(p<0.001)$. The interitem correlations (rho) for RV ranged between $0.044(p=0.766)$ and 0.739 $(p<0.001)$ with 7 correlations of 15 not being significant. The interitem correlations (rho) for BN ranged between $0.071(p=0.636)$ and $0.689(p<0.001)$ with 8 correlations of 15 not being significant. The interitem correlations (rho) for AV ranged between $0.216(p=0.141)$ and 0.639 $(p<0.001)$ with 1 correlation of 15 not being significant. $n$ in the correlations ranged between 39 and 48 .

Cronbach's alphae for the global scale and the four subscales were found to be between acceptable and good ${ }^{18}$ : global scale (0.864), EC (0.803), RV (0.728), BN (0.702), and $\mathrm{AV}$ (0.749). To estimate the contribution of each item to the internal consistency of the global scale, Cronbach's $\alpha$ was recalculated after deleting one item. Cronbach's $\alpha$ if item deleted decreased for all items except for items $1,9,11$, and 16 where Cronbach's $\alpha$ increased slightly $(0.865,0.874$, 0.865 , and 0.869 , respectively). To estimate the contribution of each item to the internal consistency of each subscale, Cronbach's $\alpha$ for each subscale was recalculated after deleting one item (see - Table 2). Cronbach's $\alpha$ if item deleted did not increase for subscales EC and BN. Cronbach's $\alpha$ increased if item 9 or 11 was deleted from the RV subscale and item 3 was deleted from the AV subscale. This suggests that internal consistency of the RV and AV subscales may improve with the deletion of these items. The item-total correlations were made for the global scale and separately for each subscale. The correlations (range rho 0.086-0.709) for the global scale indicated that the items contributed to the global scale with six exceptions: items 1 (rho $=0.257), 3($ rho $=0.189), 9$ (rho=0.086), $11 \quad(\mathrm{rho}=0.259), 16 \quad(\mathrm{rho}=0.105)$, and 19 (rho $=0.244$ ) had values below 0.3. ${ }^{18}$ The correlations (rho ranged between 0.311 and 0.729 ) made separately for each subscale (see - Table 2 ) indicated that the items contributed to their subscales with one exception: item 9 (rho 0.181) in the RV subscale.
Kaiser-Meyer-Olkin measure of sampling adequacy (0.630) indicated that the suitability for principal components analysis (PCA) was mediocre, while Bartlett's test of sphericity (approximate $\operatorname{chi}^{12}[276]=548.1, p<0.001$ ) indicated that the data was suitable for PCA. Based on this analysis, explorative PCA was conducted using Varimax rotation. Mean substitution was used in case of a missing value. One substitution was done in seven items (items 4, 6, 11, 15, 17, 23, and 24), two substitutions in one item (item 22), and seven substitutions in one item (item 21). The number of components extracted was based on parallel analysis, that is, Monte Carlo simulations that generate a 95th percentile cutoff line for eigenvalues which in turn is used to identify components that are not dependent on chance. ${ }^{19}$ The PCA with parallel analysis yielded three components that together accounted for $48.1 \%$ of the total variance. The component loadings for the separate items after rotation are shown in - Table 3 where components below 0.4 are suppressed. The items from the RV subscale fail to load as a separate component: three items yielded loadings that placed them in the same component as all the items in the EC subscale (component 1), and one item had loading that placed it in the same component as all the items in the BN subscale (component 3 ) but two of the subscale items yielded loadings that failed to reach the 0.4 cutoff (items 9 and 21). The items from the AV subscale loaded on the one and the same component (component 2).

\section{Test-Retest Reliability}

In the subsample of participants, intraclass correlation coefficients, Cronbach's $\alpha$, and Wilcoxon signed-rank test with Bonferroni correction were used to examine the test-retest reliability of the global scale and the separate subscales (see - Table 4). The average intraclass correlation coefficients and their 95\% confidence intervals suggest moderate to excellent test-retest reliability for subscales EC, RV, and BN and good to excellent test-retest reliability for the global scale and subscale AV. ${ }^{20}$ Cronbach's $\alpha$ indicated acceptable (subscale BN), good (global scale and subscales EC and RV), and excellent (subscale AV) internal consistency. The average differences between the two sessions ranged between -0.4 
Table 3 The items loading after Varimax rotation on the separate components with eigenvalues greater that 95th percent in the explorative PCA

\begin{tabular}{|c|c|c|c|}
\hline \multirow[t]{2}{*}{ Items } & \multicolumn{3}{|c|}{ PCA component } \\
\hline & 1 & 2 & 3 \\
\hline \multicolumn{4}{|l|}{ EC subscale } \\
\hline 4 & 0.683 & - & - \\
\hline 10 & 0.797 & - & - \\
\hline 12 & 0.657 & - & - \\
\hline 14 & 0.653 & - & - \\
\hline 15 & 0.635 & - & - \\
\hline 23 & 0.713 & - & - \\
\hline \multicolumn{4}{|l|}{ RV subscale } \\
\hline 2 & 0.725 & - & - \\
\hline 5 & 0.788 & - & - \\
\hline 9 & - & - & - \\
\hline 11 & - & - & 0.750 \\
\hline 18 & 0.818 & - & - \\
\hline 21 & - & - & - \\
\hline \multicolumn{4}{|l|}{ BN subscale } \\
\hline 1 & - & - & 0.482 \\
\hline 6 & - & - & 0.545 \\
\hline 7 & - & - & 0.519 \\
\hline 16 & - & - & 0.544 \\
\hline 19 & - & - & 0.778 \\
\hline 24 & - & - & 0.487 \\
\hline \multicolumn{4}{|l|}{ AV subscale } \\
\hline 3 & - & 0.523 & - \\
\hline 8 & - & 0.640 & - \\
\hline 13 & - & 0.685 & - \\
\hline 17 & - & 0.789 & - \\
\hline 20 & - & 0.750 & - \\
\hline 22 & - & 0.646 & - \\
\hline Variance explained (\%) & 22.5 & 13.3 & 12.2 \\
\hline
\end{tabular}

Abbreviations: AV, aversiveness; BN, background noise; EC, ease of communication; RV, reverberation; PCA, principal components analysis.

Note: Also shown for each component is the amount of variation that each component accounts for. Only component loadings higher than 0.4 are shown for facilitating interpretation $(n=48)$.

and 4.1 units for the different subscales. As shown in the table, the differences were not significant prior to correction for multiple tests. These findings together suggest high testretest reliability.

\section{Differences and Associations}

Differences in APHAB scores between male and female participants are shown in - Fig. 1. Using Mann-Whitney $U$ tests on the global scale and the subscales, no significant differences were seen after corrections for multiple tests

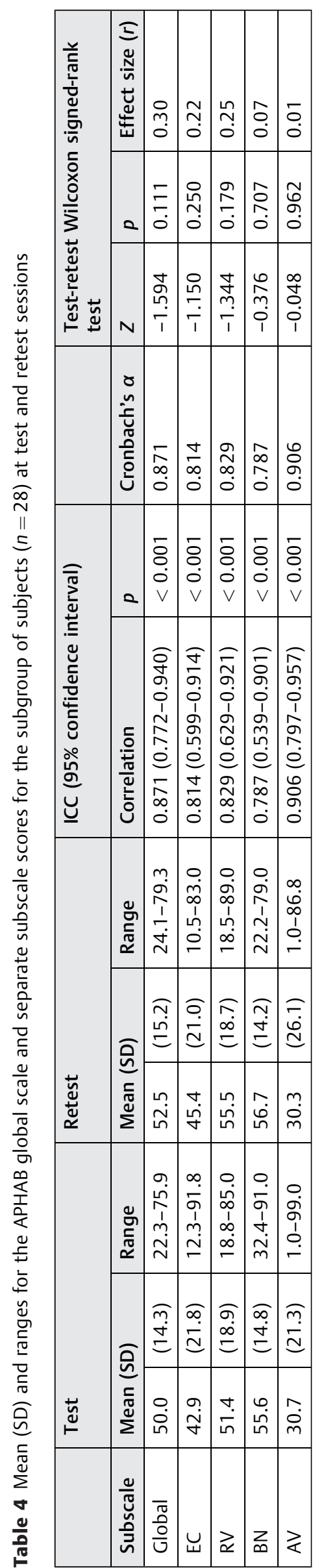




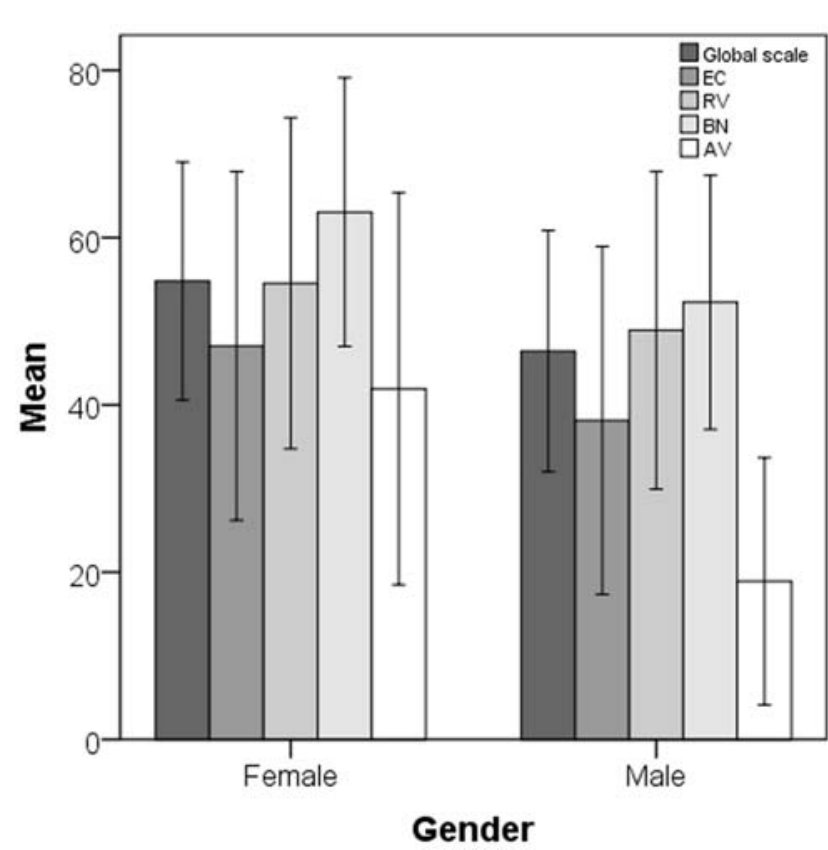

Fig. 1 Average Abbreviated Profile of Hearing Aid Benefit (APHAB) global scale and subscale scores for male $(n=31)$ and female participants $(n=17)$. Error bars denote \pm 1 standard deviation.

between male and female participants in APHAB scores on the global scale $(U[46]=181, p=0.75, r=0.26)$ or on subscales EC $(U[46]=1.197, \quad p=0.152, \quad r=0.21), \quad \mathrm{RV} \quad(U$ $[46]=220, p=0.348, \quad r=0.14)$, and $\mathrm{BN}(U[46]=165.5$, $p=0.035, r=0.31$ ), although intermediate and large effect sizes were seen for the global scale and subscales EC and BN. A significant difference was seen between male and female participants in scores on subscale AV $(U[46]=100.5$, $p<0.001, r=0.51)$ after correction for multiple tests. No significant differences were seen in PTA $_{\text {best }}(U[46]=194.5$, $p=0.136, r=0.21)$ or $\mathrm{PTA}_{\text {worst }}(U[46]=212, p=0.266$, $r=0.16$ ) between males and females.

Differences in scores on the APHAB global scale and subscales were examined between different types of hearing loss (see - Table 1) using Kruskal-Wallis test. No significant effects were seen between the three types of hearing loss for the global scale (chi-square $=3.141, p=0.208, \eta^{2}{ }_{H}=0.03$ ) or subscales EC (chi-square $=5.720, p=0.057, \eta^{2}{ }_{\mathrm{H}}=0.08$ ), RV (chi-square $=2.439, p=0.295, \eta^{2}{ }_{H}=0.01$ ), and BN (chisquare $\left.=0.003, p=0.999, \eta^{2}{ }_{H}=-0.04\right)$. A significant main effect was seen for subscale AV (chi-square $=9.538$, $\left.p=0.008, \eta^{2}{ }_{\mathrm{H}}=0.17\right)$. Bonferroni-corrected post hoc analysis using Mann-Whitney $U$ tests showed that the AV subscale scores for those with monaural mixed hearing loss was significantly higher than for those with bilateral sensorineural hearing loss $(p=0.005, r=0.46)$ but not than for those with mixed binaural hearing loss $(p=0.229, r=0.21)$. Scores on the AV for the group with mixed binaural hearing loss were not significantly different from those for the group with binaural sensorineural hearing loss after correction $(p=0.35, r=0.35)$. Using Kruskal-Wallis test, differences in PTA $_{\text {best }}$ and PTA worst $_{\text {between the groups with different }}$ types of hearing loss were examined (see - Table 1). Signifi- cant effects were seen between the groups for both PTA best $_{\text {b }}$ (chi-square $=25.355, p<0.001, \eta^{2}{ }_{H}=0.52$ ) and PTA worst (chi-square $=15.934, p<0.001, \mathrm{n}_{\mathrm{H}}^{2}=0.31$ ). For PTA $\mathrm{P}_{\text {best }}$ Bonferroni-corrected post hoc analysis showed significant differences between the groups with monaural mixed hearing loss and those with binaural mixed hearing loss $(p<0.001$, $r=0.85$ ) and those with binaural sensorineural hearing $\operatorname{loss}(p<0.001, r=0.77)$ but not between those with binaural mixed hearing loss and binaural sensorineural hearing loss $(p=0.512, r=0.11)$. For PTA worst $_{\text {Bonferroni-corrected post }}$ hoc analysis showed significant differences between the groups with monaural mixed hearing loss and those with binaural mixed hearing loss ( $p=0.001, r=0.65)$ but not those with binaural sensorineural hearing loss $(p=0.288$, $r=0.18$ ). A significant difference was seen between those with binaural mixed hearing loss and binaural sensorineural hearing loss $(p<0.001, r=0.61)$.

Spearman's rank correlation coefficients (rho) were calculated to assess the association between age, PTA $\mathrm{best}_{\text {, }}$ PTA $\mathrm{A}_{\text {worst }}$, and scores on the global scale and the subscales. A strong positive correlation was seen between age and PTA $A_{\text {best }}$ (rho $[46]=0.607, p<0.001$ ) but not between age and PTA worst $_{\text {(rho }}$ $[46]=0.108, p=0.465)$. Age showed a significant positive correlation with the global scale $(\mathrm{rho}[46]=0.289, p=0.047$ ) and with the EC subscale ( $\mathrm{rho}[46]=0.370, p=0.010$ ). Age was not significantly correlated with the RV, BN, or AV subscales (rho[46] $< \pm 0.280, p \geq 0.054$ ).

PTA $_{\text {best }}$ showed significant positive correlations with the global scale (rho[46] $=0.310, p=0.032$ ), subscales EC (rho $[46]=0.414, p=0.003)$, and $\mathrm{RV}(\operatorname{rho}[46]=0.367, p=0.010)$ and a significant negative correlation with subscale AV (rho

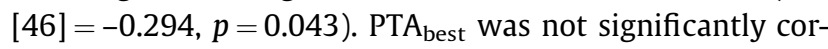
related with the subscale $\mathrm{BN}$ ( $\mathrm{rho}[46]=-0.049, p=0.741$ ). PTA $_{\text {worst }}$ showed significant positive correlations with the global scale $(\mathrm{rho}[46]=0.389, p=0.006)$ and subscales EC $(\mathrm{rho}[46]=0.395, \quad p=0.005) \quad$ and $\mathrm{RV} \quad(\mathrm{rho}[46]=0.407$, $p<0.004)$. PTA $A_{\text {worst }}$ was not significantly correlated with the subscale $\mathrm{BN}(\mathrm{rho}[46]=0.047, p=0.752$ ) or AV (rho $[46]=0.141, p=0.338)$.

\section{Discussion}

The present study evaluated disability in everyday life associated with a hearing loss in patients seeking audiological rehabilitation for the first time using a Swedish translation of the APHAB. This was done by exploring the psychometric properties of the questionnaire and how age, gender, type of hearing loss, and degree of hearing loss influence outcomes. The psychometric properties indicate high test-retest reliability but it seems to be some potential issues with the properties of the RV subscale. With few exceptions APHAB scores were not influenced by age, gender, or type of hearing loss. APHAB scores were generally influenced by degree of hearing loss in both the best and the worst ear.

\section{Psychometric Properties and Test-Retest Reliability} The present average scores on the APHAB global scale and subscales for all subjects were generally lower compared 
with previous reports. ${ }^{4,9,12}$ In a similar sized sample, Cox and Alexander ${ }^{4}$ examined slightly older participants (but with similar age range) and reported higher scores for all subscales except for the AV subscale where the scores were similar to the present study. Their participants were experienced hearing aid users with between 6 weeks' and more than 10 years' experience. Arlinger et al ${ }^{12}$ examined slightly younger hearing aid users with several years' experience and reported higher average scores on all subscales except for the AV subscale where they found a similar average score as in the present study. Cox et $\mathrm{al}^{9}$ reported APHAB subscale scores for a slightly larger sample of experienced hearing aid users with bilateral symmetric sensorineural hearing loss ranging from mild to moderate-severe in their best ear. The present average subscale scores for all participants were very similar to those reported for those with moderate hearing loss in Cox et $\mathrm{al}^{9}$ with the exception for the AV subscale where the present average was lower. It is possible that the participants in the previous studies who have experienced the benefits of using their hearing aids may rate that their unaided hearing results in more activity limitations and participation restrictions than the present participants who have no experience with using hearing aids. This argument finds some support in the study by Berninger and Karlsson. ${ }^{13}$ They examined subjects with no previous hearing aid experience and found average subscale scores that closely resembles the average scores of the present study. On the other hand, Heggdal et $\mathrm{al}^{11}$ found no difference between experienced and firsttime hearing aid users on an additionally abbreviated version of the APHAB. Due to the differences between their version of the APHAB and the original used in the present study it is hard to draw any conclusions.

The present analyses of the psychometric properties indicate that not all items in the global scale were significantly intercorrelated. This may be expected since the global scale represents a combination of scores from three different subscales (composite score of subscales EC, RV, and BN). Cronbach's $\alpha$ indicated that internal consistency for the global scale was good which suggests that the items in the global scale seem to measure the same basic construct. The basic construct can be hypothesized to be disability in everyday life associated with a hearing loss. APHAB may help a patient to take more active part in their own rehabilitation, by uncovering which areas of sound perception is more affected than others. On the flipside of disability in everyday life, lies ability or participation in everyday life. An individual's poor (high) score on APHAB may indicate poor ability in everyday activities according to the ICF model. ${ }^{8} \mathrm{APHAB}$ may be a support in rehabilitation planning according to ICF. The deletion of the items $1,9,11$, and 16 improved the internal consistency of the global scale, although not to any larger extent. The homogeneity of the items was also assessed using interitem correlations. They indicated that all items contributed to the global scale except items 1, 9, and 16 . Notably, these items are three of six items with a reverse response order. All but six APHAB items have a negative statement, for example, in item 2 "I miss a lot of information when I'm listening to a lecture" where a higher rating on the paper (not the scoring) is indicating less problems. However, positive statements are used for the reversed items (items 1,9 , $11,16,19$, and 21) a higher rating on the paper (not the scoring) indicates more problems, for example, item 1 "When I am in a crowded grocery store, talking with the cashier, I can follow the conversation." It is possible that this have influenced the responses in such manner that these items do not contribute to the scale. Based on this, it seems possible to improve the psychometric properties of the APHAB global scale by deleting items 1, 9, 11, and 16 from this score.

Not all items in a subscale were significantly correlated with the other items in that subscale. This is unexpected as we would expect homogeneity within items representing a separate subscale. However, the item-total correlations for each subscale indicated homogeneity for all items except one (item 9). The internal consistency of the four separate subscales was found to range between acceptable and good. This suggests that the items in a subscale seem to represent a single basic construct. However, the Cronbach's alphae for the four subscales are lower $(\sim 0.07-0.12)$ than those reported by Cox and Alexander ${ }^{4}$ who reported values above 0.8 for all subscales in a slightly larger sample. In the present study, the deletion of one or a few items improved the internal consistency of the RV, BN, and AV subscales. However, the improvements in Cronbach's alphae after deletion were not very large. It is possible that a larger sample size would have made these changes even smaller.

The explorative PCA indicated that the items loaded on three components. We would expect that the items would be organized into four components where the items in each subscale loaded on a separate component that represented its subscale. Previous studies do not report PCA component loadings for the original $\mathrm{APHAB}$ questionnaire. In the present study, the items of RV subscale failed to load as a separate factor. The RV subscale items loaded on either the same components as the EC subscale items or the BN subscale items. This suggests that there is an overlap in constructs with the EC and BN subscales in the constructs that the items in the RV subscale measure. A couple of items in the RV subscale did not reach sufficient loadings to be reliably placed in a component. This suggests that the combination of the subscales EC, RV, and BN into a global scale is reasonable but that there might be some difficulties in interpretation when using the RV items as a separate subscale.

In the present study, the test-retest reliability measured using the unaided $A P H A B$ was found to be moderate to excellent for the APHAB global scale and the four subscales. This is a finding similar to a previous study. ${ }^{4}$ The slight changes in the average scores between the two assessments in the present study are similar to those reported previously. ${ }^{4,12}$

\section{Differences and Associations}

The present findings suggest that APHAB global scale and subscale scores are not generally influenced by gender in subjects without experience of using hearing aids. However, the general trend is that the male scores are lower for all measures. The difference was significant for the AV subscale and medium to large effect sizes were seen for the global scale and the EC and BN subscales. It is possible that the 
significantly lower AV subscale scores for male participants may have been influenced by the fact that they also have slightly poorer best and worst ear hearing (although not significantly but with moderate effect sizes). Previous studies do not report gender differences for the original APHAB, although Heggdal et $\mathrm{al}^{11}$ using an additionally abbreviated version of the APHAB found no differences between male and female participants as in the present study.

The present findings indicate that the type of hearing loss did not influence scores on the APHAB global scale and subscales, although with one exception: the scores on the $\mathrm{AV}$ subscale were significantly higher for those with monaural mixed hearing loss than those with binaural sensorineural hearing loss. As participants with monaural mixed hearing loss also had significantly better PTA best $_{\text {, this could }}$ indicate that AV toward environmental sounds may deteriorate with better hearing. This is a finding similar to previous investigations. When hearing aids are fitted, the scores on the AV subscale often increases. ${ }^{12,13}$ This indicates that improved audibility for environmental sounds are related to increased AV toward environmental sounds. When comparing with normal hearing participants as reported by Cox and Alexander, ${ }^{4}$ the present average $\mathrm{AV}$ subscale score lies below the 95th percentile value (55). However, in comparison with these participants with normal hearing the present scores on the other subscales (EC, RV, and BN) are all above the 95th percentile values indicating that monaural mixed hearing loss seems to have an impact on self-reported disability in everyday life associated with a hearing loss.

The degree of hearing loss in the better as well as the worse ear was significantly associated with the global scale, $\mathrm{EC}$, and RV subscale scores. Cox et $\mathrm{al}^{9}$ found medium to large positive correlations between scores on the subscales EC, RV, and BN for three different measures of best ear PTA in experienced hearing aid users. They did not report associations between PTA and the global scale. It is unclear why we found no significant correlation between PTA $A_{\text {best }}$ or PTA $\mathrm{A}_{\text {worst }}$ and the BN subscale scores. One possibility is that, again, the participants in the present study had no hearing aid experience. It is also possible that the use of participants with three different types of hearing loss may have influenced this finding. The associations between the PTA measures and the different APHAB scales may differ depending on type of hearing loss. However, the three groups used in the present study are too small to be able to test this hypothesis. Future studies with larger samples are required. Furthermore, in the study by Cox et al, ${ }^{9}$ no association between PTA and the AV subscale was found. This is contrary to the present findings where PTA $\mathrm{B}_{\text {best }}$ showed a significant negative correlation with the AV subscale. They explained that AV was not associated PTA by the fact that the AV subscale relates to unpleasantness of environmental sounds while PTA relates to detecting soft sounds. ${ }^{9}$ It is unclear why we found this association but the interpretation is that participants with better ear hearing report higher scores on the AV subscale. This seems reasonable and, as previously stated, in line with previous findings that improved audibility for environmental sounds is related to increased AV toward environmental sounds. ${ }^{12}$

\section{Conclusion}

The present study showed that the psychometric properties of this Swedish version of the APHAB can be improved by addressing some inconsistencies found in the RV subscale. The items from the RV subscale failed to load as a separate component and the internal consistency of the subscale improved by removing a couple of items. Despite this, the findings suggest high test-retest reliability. The age, gender, and type of hearing loss do not have a clear impact on APHAB scores but, as reported previously, the degree of hearing loss scores do.

\section{Authors' Contributions}

The authors alone are responsible for the content and writing of the article. The authors confirm this manuscript is an original contribution, not previously published and not under consideration for publication elsewhere.

\section{Notification of Ethical Adherence}

The study received approval from the regional ethics committee. The study adhered to the Declaration of Helsinki ethical principles.

\section{Conflict of Interest}

None.

\section{References}

1 Arlinger S. Negative consequences of uncorrected hearing loss-a review. Int J Audiol 2003;42(Suppl 2):S17-S20

2 Stark P, Hickson L. Outcomes of hearing aid fitting for older people with hearing impairment and their significant others. Int J Audiol 2004;43:390-398

3 Öberg M, Lunner T, Andersson G. Psychometric evaluation of hearing specific self-report measures and their associations with psychosocial and demographic variables. Audiol Med 2007; 5:188-199

4 Cox RM, Alexander GC. The abbreviated profile of hearing aid benefit. Ear Hear 1995;16:176-186

5 Beck LB. The role of outcomes data in health-care resource allocation. Ear Hear 2000;21:89S-96S

6 Knudsen LV, Oberg M, Nielsen C, Naylor G, Kramer SE. Factors influencing help seeking, hearing aid uptake, hearing aid use and satisfaction with hearing aids: a review of the literature. Trends Amplif 2010;14:127-154

7 Hickson L, Clutterbuck S, Khan A. Factors associated with hearing aid fitting outcomes on the IOI-HA. Int J Audiol 2010;49:586-595

8 WHO. International Classification of Functioning Disability and Health: ICF. Geneva: World Health Organization; 2001

9 Cox RM, Alexander GC, Gray GA. Audiometric correlates of the unaided APHAB. J Am Acad Audiol 2003;14:361-371

10 Löhler J, Wollenberg B, Schlattmann P, Hoang N, Schonweiler R. Associations between the probabilities of frequency-specific hearing loss and unaided APHAB scores. Eur Arch Otorhinolaryngol 2017;274:1345-1349

11 Heggdal POL, Nordvik O, Brännström J, Vassbotn F, Aarstad AK, Aarstad HJ. Clinical application and psychometric properties of a Norwegian questionnaire for the self-assessment of communication in quiet and adverse conditions using two revised APHAB subscales. J Am Acad Audiol 2018;29:25-34

12 Arlinger S, Billermark E, Öberg M, Lunner T, Hellgren J. Clinical trial on a hearing aid. Scand Audiol 1998b27:51-61 
13 Berninger E, Karlsson KK. Clinical study of Widex Senso on firsttime hearing aid users. Scand Audiol 1999;28:117-125

14 IEC 60318-3 IEC 60318-3. Electroacoustics - Simulators of human head and ear- Part 3: acoustic coupler for the calibration of supraaural earphones used in audiometry. Geneva: International Electrotechnical Commission; 1998

15 ISO 389-3. ISO 389-3 Acoustics: reference zero for the calibration of audiometric equipment. Part 3: reference equivalent threshold force levels for pure tones and bone vibrators. Geneva: International Organization for Standardization; 1994

16 ISO 389-1 ISO 389-1. Acoustics: reference zero for the calibration of audiometric equipment. Part 1: reference equivalent threshold sound pressure levels for pure tones and supra-aural earphones. Geneva: International Organization for Standardization; 1998

17 ISO 8253-1 ISO 8253-1. Acoustics: audiometric test methods part 1: basic pure tone air and bone conduction threshold audiometry. Geneva: International Organization for Standardization; 1998

18 Kline P. A Psychometrics Primer. London: Free Association Books; 2000

19 Wood ND, Akloubou Gnonhosou DC, Bowling J. Combining parallel and exploratory factor analysis in identifying relationship scales in secondary data. Marriage Fam Rev 2015;51:385-395

20 Koo TK, Li MY. A guideline of selecting and reporting intraclass correlation coefficients for reliability research. J Chiropr Med 2016;15:155-163 